\title{
Modelling of Electrical Power Distribution Systems by Application of Experimental Design
}

\author{
Joanicjusz Nazarko, Member, IEEE \\ Division of Applied Informatics \\ University of Finance and Management, Bialystok, Poland \\ jnazarko@cksr.ac.bialystok.pl
}

\begin{abstract}
The purpose of this paper is to demonstrate the application of the method of experimental design to the analysis of electrical power distribution systems. The theory of experimental design allows us to construct and experimentally verify the qualitative model of a power distribution system in order to analyse a significance of each component in distribution system modelling. The paper presents results obtained from simulation studies on the effect of uncertain input data on the calculation of output characteristics of power distribution systems. An investigation of input data errors and their influence on systems analysis results has a particular importance in real-time modelling of power systems. The simulation experiment was carried out for the numerical model of an existing radially operated power distribution system of $15 \mathrm{kV}$.
\end{abstract}

Key Words: Power Distribution Systems, Experimental Design, Mathematical Modelling, Data Errors

\section{INTRODUCTION}

The purpose of this paper is to demonstrate the application of the method of experimental design to the analysis of electrical power distribution systems. Most experiments are of exploratory nature: investigator is interested in finding out what factors have an influence on the outcome of a certain process [3].

A study method assuming the representation of the studied object as a system which transforms the reaction of the environment on the object (input variables) into the system answer (output variables) is known as the system analysis. Output signals are created by the object as a result of technological and organisational processes that proceed in the object (Fig. 1).

Physical phenomena occurring in a power distribution system during distribution and utilisation of electrical energy are caused by many different processes proceeding in the system and its environment. Quantitative description of the phenomena occurring in the system by analysis of all possible relationships between these phenomena and processes is, in practice, impossible. The principles describing the behaviour of random phenomena may be determined by probabilistic and statistical analysis only [9].

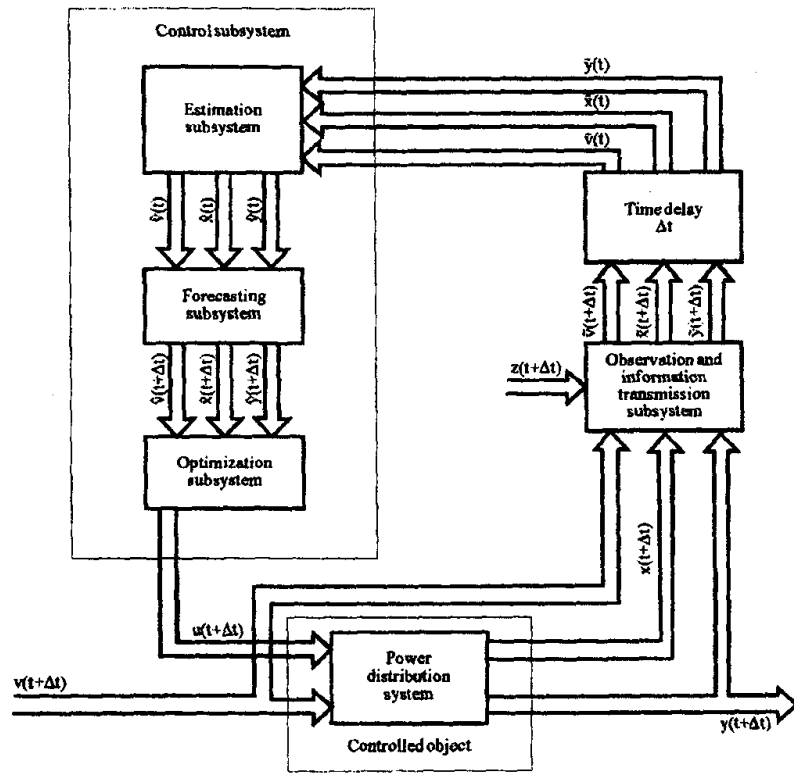

Fig. 1. The diagram of the information structure of electrical power distribution systems

A major tool for analysing the performance of power systems are load flow computations. They involve evaluation of active and reactive power flows in the system elements and of bus voltages. For operational engineers, power flow is the basis for system calculations and for optimisation of system control. For planning engineers, power flow influences various factors such as transformer and cable ratings, capacitor bank requirements, and peak load demand.

A load flow in a radial distribution system is a function of system components parameters, bus loads, and voltage at supplying bus $[5,8]$.

Mathematical models are base as well for power distribution system calculations and optimisation as for system control.

Distribution system components are modelled by their equivalent circuits in terms of resistance, reactance, conductance, and susceptance. Under balanced conditions distribution system can be represented by single phase models. Feeder reactors, shunt capacitors and connectors are usually modelled by two-terminal network. Overhead and cable lines are represented by $\pi$-equivalent circuits. Transformers are modelled by $\Gamma$-equivalent circuits. The component interconnections constitute the equivalent circuit of a distribution system. Loads are normally specified by 
their active and reactive power requirements, assuming they are unaffected by the small variations of voltage and frequency during normal steady-state operation.

Taking into consideration the above mentioned circumstances the power flow in a radially operated system can be calculated with satisfactory accuracy for practical implementation from the following relationship $[5,8]$ :

$$
\underline{\mathbf{S}}=\mathbf{D}^{T} \underline{\mathbf{S}}
$$

where:

$\underline{\mathbf{S}}-\mathbf{n} \times 1$-dimensional vector of complex power flow in system branches,

$\underline{\mathbf{S}}-\mathbf{n} \times 1$-dimensional vector of complex power received at system buses,

D- $\mathbf{n} \times \mathbf{n}$-dimensional branch-path incidence matrix, $\mathrm{n}$ - the number of system buses (without the root node).

It is evident from (1) that power flow in system branches is a linear combination of loads received at the system buses.

To ensure required precision of the load flow solution calculations are made in an iterative way.

Models constructed in this way are the base for distribution system analysis and calculations. They have satisfying accuracy and errors caused by assumed representation are practically insignificant $[5,8]$.

The main difficulty in operational practice is accurate determination of values of system components parameters and load at receiving buses.

Errors in the determination of the parameters of the equivalent circuits primarily result from omission of the influence of weather conditions, load current, quality of materials, performance and assembly, and from differences between real and rating data.

The load at the buses of the power distribution system varies in time as a result of both periodic changes of human activities and the influence of many factors which may be considered to be random phenomena. The acquisition of load data is complex because of a large number of distribution nodes and a large area over which they are scattered. As a rule, receiving nodes are not equipped with permanent measuring instruments so measurements of loads are performed sporadically only $[7,8]$.

This paper presents results obtained from simulation studies on the effect of uncertain input data on the calculation of output characteristics of power distribution systems. A qualitative model of a power distribution system is also presented.

The next section presents theoretical background of the design of experiments. The simulation experiment designed to analyse a significance of each component in distribution system modelling is described in Section 3. Section 4 presents outcomes of statistical analysis of experiment results and the resultant qualitative model of a power distribution system. Conclusions and future directions are presented in Section 5.

\section{DESIGN OF EXPERIMENTS}

Design of the experiment is a procedure of selection of a number and conditions of realisations of experiments that are necessary and sufficient for solution a stated problem with required accuracy [3].

To perform the experiment several steps have to be made [3]:

- formulation of questions and hypotheses,

- formulation in terms of a subject matter model,

- translation into a statistical model,

- development of the statistical design,

- performance of the experiment,

- collection of data,

- statistical analysis,

- interpretation of results.

For the formal problem statement the following sets are defined $[2,3]$ :

- Set of input quantities

$$
\mathbf{X},\left\{\mathrm{X}_{\mathrm{k}}: \mathbf{k}=1,2, \ldots, \mathrm{i}\right\}
$$

where $i$ is a number of input quantities.

- Set of output quantities

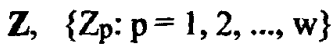

where $w$ is a number of output quantities.

A qualitative mathematical model of investigated object is introduced in the following form:

$$
F\left(X_{1}, X_{2}, \ldots, X_{i}, Z_{1}, Z_{2}, \ldots, Z_{w}\right)=0
$$

A linear model is most often employed for experimental design.

Then the investigated object is decomposed into $w$ formal objects. Each of them is described by only one output quantity. It leads to the succeeding relations:

$$
\begin{gathered}
F_{1}\left(X_{1}, X_{2}, \ldots, X_{i}, Z_{1}\right)=0 \\
F_{2}\left(X_{1}, X_{2}, \ldots, X_{i}, Z_{2}\right)=0 \\
\vdots \\
F_{w}\left(X_{1}, X_{2}, \ldots, X_{i}, Z_{w}\right)=0 .
\end{gathered}
$$

Above decomposition is a basis to define the response function of investigated object:

$$
Z_{\mathrm{p}}=\left(\mathrm{X}_{1}, \mathrm{X}_{2}, \ldots, \mathrm{X}_{\mathrm{i}}, \mathrm{Z}_{\mathrm{w}}\right)
$$

The response function is a dependence that approximates results of observations.

Results of the observations are sets of values of an output quantity that correspond with specific arrangements of input quantities. They are determined in the following way:

$$
\mathbf{Z},\left\{\mathbf{Z}_{\mathrm{p}}^{(\mathrm{u})}: \mathrm{p}=1,2, \ldots, \mathrm{w}, \mathbf{u}=1,2, \ldots, \mathrm{n}\right\}
$$

Set of $\mathbf{n}$ arrangements of input quantities makes a scheme of experiment design: 


$$
\mathbf{X}, \quad\left\{\mathbf{X}_{\mathrm{k} / \mathrm{u}}: \mathrm{k}=1,2, \ldots, \mathrm{i}, \mathrm{u}=1,2, \ldots, \mathrm{n}\right\}
$$

where:

$\mathrm{i}$ - number of input quantities,

$\mathrm{n}$ - number of arrangements of input quantities

in investigated scheme.

There are many different schemes of experiment design. The choice of the experimental layout is determined partly by the physical conditions, partly by statistical considerations. In elimination investigations fractional double level schemes play a particular role $[3,4]$. In this experimental procedure the levels of each factors are varied and all possible level combination are considered simultaneously. This allows us to obtain information about main effects and about interactions between the various factors [3].

Elimination investigations are made only for verification of the significance of the influence of input quantities on output quantities without determination of a form of function dependence between them.

An approximate model is one in which a variable is approximated by a function of variables deemed appropriate, or merely being available.

Because of reconnaissance character of the investigations it is allowed to approximate response function by linear model:

$$
z_{p}=b_{p, 0}+b_{p, 1} x_{p, 1}+b_{p, 2} x_{p, 2}+\ldots+b_{p, i} x_{p, i}
$$

where: $b_{p, 0}, b_{p, 1}, \ldots, b_{p, i}$ are polynomial coefficients.

Unknown coefficients are calculated from the following form:

$$
b_{p, k}=\frac{1}{n} \sum_{u=1}^{n} X_{k / u} Z_{p}^{(u)},
$$

where $\mathrm{X}_{0}=1$ denotes hypothetical variable.

Input quantities are normalised to $[-1,1]$ range.

Verification of the significance of polynomial coefficients is made by means of the $t$-Student test [4]. In order to attain this it is necessary to calculate $t$-statistics for each coefficient $b_{k}$ :

$$
t\left(b_{k}\right)=\frac{\left|b_{k}\right|}{S\left(b_{k}\right)}, \quad \mathrm{k}=0,1, \ldots, \mathrm{i},
$$

where: $S\left(b_{k}\right)$ is the standard deviation of $b_{k}$ coefficient.

Verification of the significance consists in comparing significance level $\alpha_{k}$ of each statistics $t\left(b_{k}\right)$ to the assumed critical value $\alpha$.

Investigated quantity $X_{k}$ is significant if

$$
\alpha_{k} \leq \alpha,
$$

and insignificant if

$$
\alpha_{k}>\alpha \text {. }
$$

In this way, on the basis of the theory of experimental design, it is possible to construct and verify the qualitative model of a power distribution system. In the next section

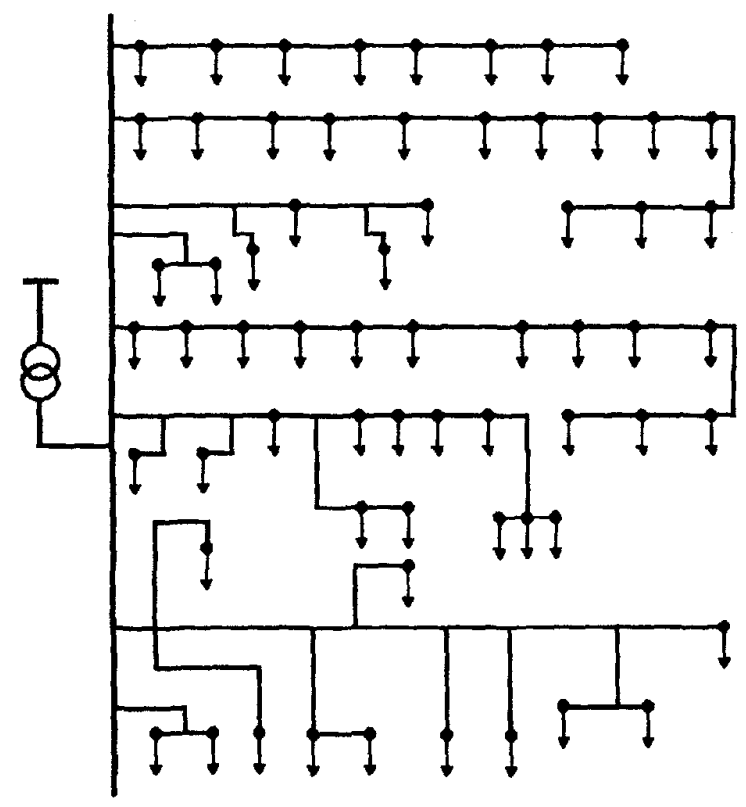

Fig. 2. The diagram of studied power distribution systems of $15 \mathrm{kV}$

the computer simulation experiment designed to analyse significance of each component in distribution systems modelling is described.

\section{FORMULATION OF THE PROBLEM}

To detect a qualitative structure of power distribution network the investigations based on experimental design procedures were performed. On the basis of physical principles of energy transmission and distribution process, 15 basic input quantities have been chosen for investigation. They represent parameters of transformers and lines, supplying bus voltage, peak active and reactive load, and power factor:

$\mathrm{X}_{1}$ - resistance of supplying transformer, $\mathrm{r}_{\mathrm{t}}$,

$X_{2}$ - reactance of supplying transformer, $X_{t}$,

$X_{3}$ - conductance of supplying transformer, $g_{t}$,

$\mathrm{X}_{4}$ - susceptance of supplying transformer, $\mathrm{b}_{t}$,

$X_{5}$ - sum of resistance of receiving transformers, $\Sigma_{r_{t}}$,

$\mathrm{X}_{6}$ - sum of reactance of receiving transformers, $\Sigma \mathrm{x}_{\mathrm{to}}$,

$X_{7}$ - sum of conductance of receiving transformers, $\Sigma g_{\text {to }}$,

$\mathrm{X}_{8}$ - sum of susceptance of receiving transformers, $\Sigma \mathrm{b}_{\text {to }}$,

$X_{9}$ - sum of resistance of lines, $\Sigma r_{l}$,

$\mathrm{X}_{10}$ - sum of reactance of lines, $\Sigma \mathrm{x}_{\mathbf{l}}$,

$X_{11}$ - sum of susceptance of lines, $\Sigma b_{l}$,

$X_{12}$ - supplying bus voltage, $u$,

$X_{13}$ - receiving transformers transformation ratio, $\vartheta$,

$\mathrm{X}_{14}$ - sum of peak active loads of receiving transformers, $\Sigma P$,

$\mathrm{X}_{15}$ - power factor, $\operatorname{tg} \varphi$. 
Power flow, power and energy loses, and voltage level are the basic functions described on network components. The following 9 basic global indices describing work conditions of power distribution systems have been chosen as output quantities for further analysis:

$\mathrm{Z}_{1}$ - active power at supplying bus, $P$,

$Z_{2}$ - reactive power at supplying bus, $Q$,

$\mathrm{Z}_{3}$ - current amplitude in supplying transformer, $\mathrm{I}$,

$\mathrm{Z}_{\mathbf{4}}$ - total active power losses, $\Delta \mathrm{P}$,

$Z_{5}$ - total load losses, $\Delta \mathbf{P}_{\mathbf{0}}$,

$\mathrm{Z}_{6}$ - total no-load losses, $\Delta \mathrm{P}_{\mathrm{j}}$,

$Z_{7}$ - daily active energy consuming by system, $A$,

$Z_{8}$ - daily active energy losses, $\Delta A$,

$Z_{9}$ - sum of square voltage deviation at the receiving buses, $\Sigma \delta \mathrm{U}^{2}$.

In order to eliminate insignificant quantities from the qualitative model of a power distribution system a special scheme of experiment was worked out. The scheme is constructed on the basis of the Plackett-Burman algorithm [2].

Assumed ranges of possible variation of input quantities are presented in Table $1[2,6,8]$.

The scheme of experiment is shown in Table 2. The sign + 'indicates the maximum value and ' $-{ }^{\prime}$ the minimum value of investigated quantity.

The central arrangements with normalised values ' 0 ' were added to the scheme to verify the permissibility of using linear model (9).
TABLE 1

\begin{tabular}{|l|r|}
\hline \multicolumn{2}{|c|}{ Ranges of possible variation of input quantities } \\
\hline Input quantities & Range of variation \\
\hline $\mathbf{r}_{\mathrm{t}}$ & $\pm 20 \%$ \\
$\mathrm{x}_{\mathrm{t}} \mathrm{g}_{\mathrm{t}}, \mathrm{b}_{\mathrm{t}}$ & $\pm 10 \%$ \\
$\Sigma_{\mathrm{t}}$ & $\pm 20 \%$ \\
$\Sigma_{\mathrm{to}}, \mathrm{g}_{\mathrm{to}}, \Sigma \mathrm{b}_{\mathrm{to}}$ & $\pm 10 \%$ \\
$\Sigma_{\mathbf{r}}$ & $\pm 12 \%$ \\
$\Sigma \mathrm{x}_{1}, \Sigma \mathrm{b}_{1}$ & $\pm 3 \%$ \\
$\mathbf{u}$ & $\pm 5 \%$ \\
9 & $\pm 10 \%$ \\
$\Sigma \mathrm{P}$ & $\pm 20 \%$ \\
$\mathrm{tg} \varphi$ & $\pm 40 \%$ \\
\hline
\end{tabular}

For each arrangement of input quantities the system calculations were performed. Computations were made under the following algorithm:

1. Power flow study and calculations of output quantities $\left(Z_{1}, \ldots, Z_{9}\right)$ for each arrangements $u$ of input quantities $\left(X_{1}, \ldots, X_{15}\right)$.

2. Calculations of polynomial coefficients for each of output quantity, according to (10).

3. Verification of the significance of polynomial coefficients using t-Student test according to (11).

Scheme of experiment

TABLE 2

\begin{tabular}{|c|c|c|c|c|c|c|c|c|c|c|c|c|c|c|c|c|}
\hline Arrangement & \multicolumn{16}{|c|}{ INPUT QUANTITIES } \\
\hline $\mathbf{u}$ & $\mathrm{x}_{0}$ & $x_{1}$ & $\mathrm{X}_{2}$ & $\mathrm{X}_{3}$ & $\mathrm{X}_{4}$ & $\mathrm{X}_{5}$ & $x_{6}$ & $x_{7}$ & $x_{8}$ & $x_{9}$ & $x_{10}$ & $x_{11}$ & $x_{12}$ & $x_{13}$ & $x_{14}$ & $x_{15}$ \\
\hline 1. & + & + & + & + & + & - & + & - & + & + & - & - & + & - & - & - \\
\hline 2. & + & + & + & + & - & + & - & + & + & - & - & + & - & - & - & + \\
\hline 3. & + & + & + & - & + & - & + & + & - & - & + & - & - & - & + & + \\
\hline 4. & + & + & - & + & - & + & + & - & - & + & - & - & - & + & + & + \\
\hline 5. & + & - & + & - & + & + & - & - & + & - & - & - & + & + & + & + \\
\hline 6. & + & + & - & + & + & - & - & + & - & - & - & + & + & + & + & - \\
\hline 7. & + & - & + & + & - & - & + & - & - & - & + & + & + & + & - & + \\
\hline 8. & + & + & + & - & - & + & - & - & - & + & + & + & + & - & + & - \\
\hline 9. & + & + & - & - & + & - & - & - & + & + & + & + & - & + & - & + \\
\hline 10. & + & - & - & + & - & - & - & + & + & + & + & - & + & - & + & + \\
\hline 11. & + & - & + & - & - & - & + & + & + & + & - & + & - & + & + & - \\
\hline 12. & + & + & - & - & - & + & + & + & + & - & + & - & + & + & - & - \\
\hline 13. & + & - & - & - & + & + & + & + & - & + & - & + & + & - & - & + \\
\hline 14. & + & - & - & + & + & + & + & - & + & - & + & + & - & - & + & - \\
\hline 15. & + & - & + & + & + & + & - & + & - & + & + & - & - & + & - & - \\
\hline 16. & + & - & - & - & - & - & - & - & - & - & - & - & - & - & - & - \\
\hline 17. & + & 0 & 0 & 0 & 0 & 0 & 0 & 0 & 0 & 0 & 0 & 0 & 0 & 0 & 0 & 0 \\
\hline
\end{tabular}

\section{RESULTANT MODEL}

The simulation experiment was carried out for numerical model of an existing $15 \mathrm{kV}$, radially operated power distribution system [8]. The system is supplied from $110 / 15 \mathrm{kV}, 16 \mathrm{MVA}$ supplying transformer and supplies 65 receiving transformers of $15 / 0.4 \mathrm{kV}$ (Fig. 2).
On the basis of statistical analysis of calculations, input quantities were divided into two groups: significant and insignificant. In the Table 3, significant input quantities for each output quantity, at the level of significance $\alpha=$ 0.05 , are presented. The input quantities are placed in the sequence from the most significant to the least significant. 
As shown, active load at system buses is the most significant factor in most computations made on power distribution systems, such as power flows $(P, Q, I)$, total active power losses $(\Delta P)$, total load losses $\left(\Delta P_{0}\right)$, energy consumption (A), and energy losses $(\triangle A)$. However, in assumed range of input quantities variation this factor may be assented insignificant in computations of no-load losses $\left(\Delta \mathrm{P}_{\mathrm{j}}\right)$ and sum of square voltage deviation at the receiving buses $\left(\Sigma \delta \mathrm{U}^{2}\right)$.

In order to properly analyse the performance of distribution circuits, it is essential to obtain accurate estimates of power consumption. The appropriate evaluation of loads at the system busses is the most important part of the system analysis.

The experiment has shown that in assumed range of input quantities variation it is allowed to use linear model for approximation of response function for all investigated output quantities.

TABLE 3

Significant input quantities $(\alpha=0.05)$

\begin{tabular}{|c|l|}
\hline Output quantity & \multicolumn{1}{|c|}{$\begin{array}{c}\text { Significant input } \\
\text { quantities }\end{array}$} \\
\hline $\mathbf{P}$ & $\Sigma \mathrm{P}$ \\
$\mathbf{Q}$ & $\Sigma \mathrm{P}, \mathrm{tg} \varphi, \Sigma \mathrm{b}_{\mathrm{l}}$ \\
$\mathbf{I}$ & $\Sigma \mathrm{P}, \mathrm{u}$ \\
$\Delta \mathrm{P}$ & $\Sigma \mathrm{P}, \mathrm{u}, \Sigma \mathrm{g}_{\mathrm{to}}$ \\
$\Delta \mathrm{P}_{\mathrm{o}}$ & $\Sigma \mathrm{P}, \mathrm{u}, \Sigma \mathrm{r}_{\text {to }}$ \\
$\Delta \mathrm{P}_{\mathrm{j}}$ & $\mathrm{u}, \mathrm{9}, \Sigma \mathrm{g}_{\mathrm{to}}$ \\
$\mathrm{A}$ & $\Sigma \mathrm{P}$ \\
$\Delta \mathrm{A}$ & $\Sigma \mathrm{P}, \Sigma \mathrm{g}_{\text {to }}, \Sigma \mathrm{r}_{\text {to }}$ \\
$\Sigma \delta \mathrm{U}^{2}$ & $\mathrm{9}, \mathrm{u}$ \\
\hline
\end{tabular}

\section{CONCLUSIONS}

On the basis of statistical analysis of the results of the specially designed computer experiment the following conclusions were formulated:

1) The theory of experimental design allows us to construct and experimentally verify the qualitative model of power distribution system that is a base for system optimisation and control.

2) Proper evaluation of active loads at system buses is the most important point in power distribution system modelling.

3) Errors in load evaluation existing today in operational practice have a fundamental effect on the accuracy of computations made on system.

It seems that future investigations concerning power distribution system automation should be concentrated on proper load estimation algorithms. Load estimates are the most vital data requirements in network planning and operation $[1,6,8,10]$.

\section{ACKNOWLEDGMENTS}

This work is partly supported by the State Committee for Scientific Research (KBN) under contract W/ZZM/1/99.

\section{REFERENCES}

[1] Broadwater R., Sargent A., Yaraji A., Shaalan H., Nazarko J.: Estimating Substation Peaks from Load Research Data. IEEE Transactions on Power Delivery, Vol. 12, No. 1, January 1997. pp. 451-456.

[2] Gusejnov F.G., Mamadjarov O.S.: Experimental Design in Electrical Power Engineering. (In Russian). Energoatomizdat, Moscow 1988.

[3] Hinkelmamn K, Kempthome $O$.: Design and Analysis of Experiments. John Wiley \& Sons, Inc. New York 1994.

[4] Johnson N.L., Leone F.C.: Statistics and Experimental Design in Engineering and Phxical Sciences. John Wiley and Sons Ltd., New York 1977.

[5] Kujszczyk S.: Modern Methods of Power Distribution System Calculations. (In Polish). WNT, Warsaw 1984.

[6] Kujszczyk S., Nazarko J., Charytoniuk W., Broadwater RP.: The Effect of Expert Evaluations on the Efficiency of Decision Processes in Power Distribution Systems. Athens Power Tech, Joint International Conference, Athens, Greece, Sept. 5-8 1993.

[7] Morgan MG., Talugdar S.N.: Electric Power Load Management: Some Technical, Economic, Regulatory and Social Issues. Procedings of the IEEE, Vol. 67, № 2, 1979.

[8] Nazarko J.: Modeling of Electrical Power Distribution Systems. Bialystok Technical University Publisher, Bialystok 1993.

[9] Nazarko J., Cross G., Zalewski W.: The Probabilistic Approach to the Analysis of Power Distribution Systems. In: Stockholm Power Tech. Intemational Symposium on Electric Power Enginesring. Stockholm, 1995. pp. 750-755.

[10] Nazarko J., Zalewski W.: The Fuzzy Regression Approach to Peak Load Estimation in Power Distribution Systems. IEEE Transactions on Power Systems, Vol.14, No. 3, August 1999, pp. 809-814.

\section{BIOGRAPHY}

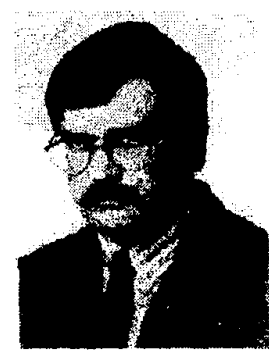

Jeanicjuse Navarko received his Ph.D. and D.Sc. degrees in Electrical Engineering from the Warsaw University of Technology in 1983 and 1992, respectively. He is currently the Professor of Electrical Engineering at the Bialystok Technical University, Poland and he serves as the head of Chair of Business Informatics and Logistics $\mathrm{He}$ is also a visiting lecturer at the Warsaw University of Technology. His research activity is centred on automation of power distribution systems with emphasis on modelling and analysis of distribution systems in uncertain conditions. Specific research areas have included load estimation, supply restoration, energy loss evaluation and power quality. He is the author of over 90 papers. He is a member of IEEE, IEE, CIGRE and SEP. 